Preprint

\title{
Moral Foundations' Methodological Foundations: A Systematic Analysis of Reliability in Research Using the Moral Foundations Questionnaire
}

\author{
Daniel J. Tamul \\ Department of Communication, Virginia Tech \\ Malte Elson \\ Department of Psychology, Ruhr University Bochum \\ James D. Ivory, Jessica C. Hotter, Madison K. Lanier, \\ and Jordan T. Wolf \\ Department of Communication, Virginia Tech \\ Nadia I. Martinez-Carrillo \\ English and Communication Studies Department, Roanoke College
}

\begin{abstract}
The Moral Foundations Theory (MFT) has drawn significant interest from the popular press and academics alike. One of its primary constituting methodologies is the Moral Foundations Questionnaire (MFQ), a self-report instrument aimed to assess the use of five sets of moral intuitions when making moral judgments. The proposed universality and moduliarity of both MFT and MFQ have been challenged conceptually and empirically. We examine the scale's development and offer a systematic content analysis of 539 scholarly works using the MFQ to estimate its overall reliability. Altogether, $62 \%$ of the studies reported Cronbach's alpha as an indicator for reliability (38\% reported no reliability estimate at all). Mean Cronbach's alpha scores for four of the five subscales were below .70. We discuss implications for the measurement of moral foundations and the evidentiary basis of Moral Foundations Theory.
\end{abstract}

Keywords: Moral Foundations Theory; Moral Foundations Questionnaire; Reliability; Content Analysis 


\section{Moral Foundations' Methodological Foundations: A Systematic Analysis of Reliability in Research Using the Moral Foundations Questionnaire}

Moral Foundations Theory (MFT) posits political polarization or a "culture war" between partisans in contemporary society can be accounted for by conservatives and liberals having respectfully cultivated binding versus individualizing moral foundations (J. Graham et al., 2009 J. Graham et al., 2011; Haidt et al., 2009 Haidt \& Joseph, 2004). In short, the theory maintains that liberals and conservatives disagree on what constitutes morality. Haidt and Graham (2007) argue that MFT advances the study of morality and moral psychology as these academic fields have only studied "a subset of human morality," namely liberal morality, while at the same time labeling conservative moralities such as in-group, loyalty, and purity as "bad" (p. 110), which precludes those moralities from rigorous study in academia. MFT also purportedly offers a social psychological account of the substantial variations and constants of morality across cultures. It proposes several innate, universal foundations or "intuitive ethics" on which cultures build unique systems of morality.

The Moral Foundations Questionnaire (MFQ), both in its original version (J. Graham et al., 2009) and in a subsequently validated form (J. Graham et al., 2011), has been used in a large number of studies and is fundamental to the theory's utility and accuracy of its predictions, including a range of popular media sources who have given MFT attention as an explanation for political "culture war" issues. MFT accounts for the inability for liberals and conservatives to engage in productive dialogue on morality is because of an inability for liberals to recognize conservative morality, but MFT can "be used as a doorway through the wall" (Haidt \& Graham, 2007, p.111). Given the evidential weight the scale is supposed to carry, it should exhibit robust psychometric properties. In this study, we examine systematically the status of reliability measurement and reporting for the moral foundations questionnaire to examine the level of reliability the MFQ demonstrates across the literature and provide researchers with a clear set of expectations regarding the MFQ's reliability in research. Such an examination of the wider literature is critical because reliability is necessary — although not sufficient — to achieve validity (Drost, 2011; Meyer, 2010. Nunnally, 1978), and increase the precision of estimates underlying inferences on the development of and differences in moral morality. It is, without a doubt, "absolutely critical to measurement" (Wrench et al., 2013, p. 230).

Correspondence concerning this manuscript should be directed to Daniel J. Tamul, Department of Communication (0311), Virginia Tech; Shanks Hall Room 146, 181 Turner Street, NW, Blacksburg, VA 24061; email: tamul@vt.edu; phone: 540-231-3610; fax: 540-231-9817.

ME is supported by the Digital Society research program funded by the Ministry of Culture and Science of North Rhine-Westphalia, Germany.

Data and supplementary materials can be accessed on the Open Science Framework https://osf.io/hsq39/. The authors declare that they have no conflicts of interest with respect to the authorship or the publication of this article. ME and JI jointly generated the idea for this study and ME, JI, and DT designed the procedure employed. JH, ML, and JW collected and coded the articles used in analysis, and DT and NMC independently coded the data for sample size and collection method. JI and DT analyzed the data. DT wrote the first draft of the manuscript with ME and JI providing critical editing.

We thank Luisa Jansen for her invaluable help with preparing the data for analysis. 
In both the MFQ's original development (J. Graham et al., 2009) and later refinement (J. Graham et al., 2011), the reported reliability of the MFQ's subscales (indicated by Cronbach's alpha) were between .39 to .70 and below what is generally considered acceptable by contemporary standards (Nunnally \& Bernstein, 1994). However, since then, no systematic assessment of the MFQ's performance and psychometric properties across the literature has been conducted. While there is the possibility that the MFQ's reliability performance more generally outstrips that in its foundational work, it is also plausible that, in general, the scale underperforms relative to such research. Here we report on an examination of the broader literature that could then serve as a relief or a warning to other scholars who may be basing their use or avoidance of the MFQ by looking to principal works.

Our work also builds on a growing body of literature that has begun to probe the validity of MFT generally and the MFQ specifically. Most importantly, its universality and modularity have been challenged both conceptually and empirically. Suhler and Churchland (2011) argue that MFT's five foundations appear somewhat arbitrary given there are other foundations that are equally plausible, yet not incorporated into MFT, and that substantial intergroup differences in those foundations have not been accounted for. These arguments are supported by a range of studies. For instance, across surveyed samples from 23 out of 27 countries, Iurino and Saucier (2020) were unable to replicate the MFQ's (supposedly universal) five-factor structure proposed by J. Graham et al. (2011). Similarly, Davis et al. (2016) observed weaker relationships between conservatism and the binding foundations in Black than in White US Americans, and overall poor fit of the five-factor structure as well as problems with scalar invariance. Gray and Keeney $(2015 \mathrm{~b})$ raise doubts as to whether the foundations can be sufficiently differentiated from one another, both theoretically and empirically (see also Gray \& Keeney, 2015a). Finally, a key claim of the MFT is that the foundations should cause-or at the very least predict-political ideology. However, Hatemi et al. (2019) found that, conversely, ideology is a much better predictor of the foundations. Taken together, these findings raise some concerns about the ability of the MFQ to serve as a valid measure for Moral Foundations, if not MFT more broadly.

\section{Moral Foundations Theory}

Relevant past work has conceptualized morality and judgment within the bounds of social justice, the work of Kohlberg (1969), or caring (Gilligan, 1982, Gilligan \& Wiggins, 1987). Turiel (1983) subsequently solidified morality as "prescriptive judgments of justice, rights, and welfare pertaining to how people ought to relate to each other" (p. 3).

For some, this form of morality is too narrow: social justice research cannot account for political conservatism and, at best, conservatism is explained as a form of self-serving bias and to "justify inequality among groups and individuals" (Jost et al., 2003, p. 430). At worst, researchers resolve their inability to rationalize conservatism within a social justice framework with contempt and derision. For example, Haidt and Graham (2007) state that at a social justice conference, a presenter dismissed conservative critiques of affirmative action with "open expressions of self-righteousness and contempt" (p. 101).

Moral Foundations Theory (MFT) seeks to rectify the inability for the field to recognize a valid basis for conservative ideology and morality by expanding the field beyond 
fairness. To expand on extant scholarship of the time, Haidt and Joseph (2004) proposed an alternative theory for how morality develops. They hypothesize across their corpus of work a universal set of moral foundations (e.g. J. Graham \& Haidt, 2012; J. Graham et al., 2012 Haidt \& Graham, 2009 Haidt et al., 2009, Haidt \& Joseph, 2008). These sets of morals are innate to the human experience, specifically that this framework is "organized in advance of experience" (Marcus, 2004, p. 40), striking a balance between nativist (see Pinker, 2002) and empiricist understandings (Elman et al., 1996) of morality formation. Haidt and Joseph (2008) argue positioning MFT as straddling these two paradigms is necessary to account for the universality of their five foundations while at the same time accounting for cultural variations or "editing" of the foundations. They argue the human mind comes hardwired to learn specific systems (Haidt \& Joseph, 2004), such as fears. There are few specific fears that are hardwired into the human brain from birth, but we are predisposed to easily build a healthy fear of spiders and snakes but not benign objects like flowers (Haidt \& Joseph, 2004). Similarly, we develop intuitive ethics, "an innate preparedness to feel flashes of approval or disapproval toward certain patterns of events involving other human beings" (p. 56). More importantly, the human brain not only comes equipped to learn these ethics, but to learn specific types of morality: harm/care, fairness/reciprocity, ingroup/loyalty, authority/respect, and purity/sanctity (Haidt \& Graham, 2007). Since the foundations premiered, moralfoundations.org has renamed the scales to care/harm, fairness/cheating, loyalty/betrayal, authority/subversion, and sanctity/degradation (Haidt, 2017).

The human mind, according to MFT, comes primed to learn these five foundations, but that experience leads some individuals to rely more heavily on certain foundations than others (Haidt \& Graham, 2007; Haidt \& Joseph, 2008), which can, at least in part, account for modern "culture wars" (Koleva et al., 2012). Although much early work on MFT proposes, defines, or tests these five foundations exclusively, Haidt and Joseph (2011) stated they are working on developing or modifying these foundations in response to criticisms of the moral foundations specifically as ad-hoc and MFT's modularity more broadly as possibly "conveniently vague and context-morphable" (Suhler \& Churchland, 2011, p. 2105).

\section{Moral Foundations Questionnaire}

The Moral Foundations Questionnaire (MFQ), one of the primary measures used in empirical research on morality and a keystone of the evidence relevant to the Moral Foundations Theory, has undergone some notable changes in its development process. We attempt to reconstruct and summarize this development process here to aid the understanding of the content analysis reported further below.

The first instance of the MFQ, though not by name, was based on studies 1 and 2 in J. Graham et al. (2009), who generated 20 items to assess general considerations relevant to moral decision making, and 23 more concrete items intended to gauge moral intuitions, designed to reflect the five moral foundations of MFT. J. Graham et al. (2009) explicitly assert that it was "not [their] intention in this article to develop or validate a scale" (p. 1041), yet this set of items quickly gained significant popularity among morality researchers. Notably, this version is often referred to as MFQ-40 (or MFQ-41, if one counts the additional item checking mischievous responding), usually with a citation of the J. Graham et al. (2009) paper (e.g. Iyer et al., 2010), although there are substantial differences between the 
43 items reported in the appendix of J. Graham et al. (2009) and the $40+1$ items in the official MFQ-40 scale that had been made available for use on MoralFoundations.org.

Between (J. Graham et al., 2009) and MFQ-40, a total of 18 items were removed, 15 new ones were added, and the wording of a further five items was changed. Those substantial changes, while based on the original Graham et al. (2009) data, are, however, only documented in the subsequent (J. Graham et al., 2011) publication, where it is argued that some of the internal consistencies were low, and that some items had weak loadings on the latent factors, were loading on the "wrong" factor, or were redundant.

J. Graham et al. (2011) then report a great effort to validate the MFQ-40 based on inter-subscale correlations and correlations with other self-report measures, reducing the number of items (and changing the wording of one item) to create the now recommended 30-item MFQ-30, as well as the 20-item short-form "MFQ-20" (a subset of the MFQ-30). Please see https://osf.io/hsq39/ for a side-by-side comparison of the items in Graham et al. (2009), and MFQ-40, MFQ-30, and MFQ-20.

\section{The Reliability of the Moral Foundations Questionnaire}

Our purpose is to examine observed reliability estimates of the Moral Foundations Questionnaire as they undergird Moral Foundations Theory. In the initial development of the MFQ, reliability of the subscales was reported with Cronbach's alpha and was poor or barely acceptable by convention with scores ranging from .39 to .76 (J. Graham et al., 2009). The validation research reported in (J. Graham et al., 2011) did shorten and refine several items, and improved Cronbach's alphas to overall still moderate levels for both the MFQ$40(.69-.86)$ and the MFQ-30 (.65-.84) in two large samples. In a small sample of college students, subscale test-retest reliabilities (Pearson correlations), approximately one month between t1 and t2, showed similar levels (.69-.82). J. Graham et al. (2011) suggest these low Cronbach's alpha levels are, perhaps, a desirable tradeoff between internal consistency and content validity - the ability for a scale to exhaustively measure a concept (Bannigan \& Watson, 2009). Yet, building a scale that samples from each aspect of a concept should result in a scale where individual items are highly interrelated (Henson, 2001).

A systematic accounting of the MFQ subscale's reliabilities is required as scale reliability is an attribute not of scales but of the implementation of those scales in a particular context (Hayes, 2005 Thompson, 2003). In other words, a scale demonstrating reliability when it was first tested or validated cannot be taken to mean the test is generally reliable. Only a systematic examination of extant research can make such a determination. Estimating the overall reliability of the MFQ would be a valuable contribution to the field for several reasons. If the scales tend to demonstrate poor reliability, researchers are at greater risk of Type II error (Niemi et al., 1986). Low reliability will reduce observed correlations between variables relative to their true correlations due to noise in what the scale measures (O. P. John \& Soto, 2007). Researchers could correct for the low reliability of the MFQ scales by correcting observed correlations for attenuation, but "the ease with which this correction is made should not be seen as a license for sloppy measurement" (O. P. John \& Soto, 2007, p. 465) because, in part, it is not clear whether the attenuated correlations are in fact underestimations of true scores (Nimon et al., 2012). Researchers employing modest 
sample sizes will likely be operating with high degrees of error in their MFQ dataset and diminished statistical power to detect relationships. Much more importantly, a field's tolerance for low reliability scores in key measures can stagnate the refinement or rejection of theory as null results are more likely to be taken as indicative of problems with measurement rather than as problems stemming from theorizing (Meier, 1994) as researchers who use poorly constructed measures prone to error can fail to correctly identify when a latent variable model is misspecified (McNeish, 2018, McNeish et al., 2018). Assessing the MFQ's reliability in the wider literature can only be a boon to the MFT as the findings from this analysis can either be used to quell criticism of the scale or as a call to improve it.

Our approach is based on a burgeoning field of research that has examined the degree to which reliability information is adequately provided in scholarship (e.g. Vacha-Haase et al., 1999). Several reviews of literature have indicated that while Cronbach's alpha is the most frequently reported indicator of reliability, a substantial portion of scholarship fails to report any reliability information whatsoever (Barry et al., 2014 Flake et al., 2017, Meier \& Davis, 1990, Reichwein Zientek et al., 2008; Vacha-Haase et al., 1999). Cronbach's alpha is a function of the number of items in a test, the average covariance between itempairs, and the variance of the total score. We expect Cronbach's alpha to be the most commonly reported indicator of reliability in the MFQ literature, despite recurring criticism that internal consistency is not the same as psychometric reliability (Sijtsma, 2009) and that other indices of internal consistency should be given preference (see McNeish, 2018).

A note on Cronbach's alpha. If social scientists truly wish to use statistical coefficients like Cronbach's alpha as indicators of a scale's suitability and not merely as rhetorical tools to aid in the publication process (see I. D. John, 1992), then such systematic examinations of their performance (even lacking other, more suitable indicators of reliability than Cronbach's alpha) in a larger body of research are both necessary and actionable. According to Flake et al. (2017), these critical examinations of scale validity are necessary because external tests of validity (e.g. predictions based on scales) presuppose structural validity (e.g. internal consistency).

Researchers may at times try to pass off low scale reliabilities as the result of short scales, but "[s]hort [scale] length does not alleviate the problems of reliability" (Schmitt, 1996, p. 352) and low-reliability scales are not without consequences for researchers. Failing to factor in the degree to which scales are unreliable can diminish the power researchers have to detect true relationships between variables, thereby biasing findings toward the null hypothesis (Hedge et al., 2018). One potential solution to this problem is to correct for attenuated variables using Spearman's (1904) approach, but errors associated with items on one scale may be correlated with either its own scores or that of another scale, meaning observed correlations may be higher or lower than true score correlations (Nimon et al., 2012). Therefore, while researchers can correct for attenuation, doing so may not always result in a more accurate representation of true correlations.

The importance of reporting reliability information for a scale deployed in a study cannot be overstated. Despite Cronbach's alpha serving as the most common indicator of reliability in the field, relatively recent scholarship has pointed out that Cronbach's alpha has some drawbacks (Raykov \& Marcoulides, 2019). For example, alpha can underestimate 
true reliability when a scale does not meet the assumptions of an essentially tau-equivalent model, which relaxes the stringent assumptions of a parallel measurement model. In a parallel measurement model all items measure the same latent variable with the same degrees of precision and error while using the same scale (see Raykov, 1997). A tauequivalent model does not assume that the item error variances are equal and an essentially tau-equivalent model additionally relaxes the assumption that the items have the same degree of precision (J. M. Graham, 2006 Raykov, 1997). When this assumption is violated, alpha tends to underestimate true reliability (Miller, 1995) and that as violations of tauequivalence increase in magnitude, the underestimation of true reliability decreases (J. M. Graham, 2006). Scholars have proposed several alternatives to alpha that can, at the very least, supplement our understanding of a scales performance such as omega (Dunn et al., 2014) or reporting a greater lower bound in conjunction with alpha (see Sijtsma, 2009).

\section{MFQ Reliability in Diverse Samples}

Investigating the MFQ's performance in the wider literature is also of value since the initial MFQ measures and subsequently revised versions are derived from (large) convenience samples of participants that self-selected to take one or more surveys hosted online by the researchers. J. Graham et al. (2011) argue these volunteers proffer a generalizable sample for validating the scale because of the sheer number of participants $(N=34,476)$. Their argument is that such a sample ( $87 \%$ white, $63 \%$ male, and $80 \%$ from the United States), even though based on convenience sample, can, due to its size, be (1) sliced by nationality to validate a five-factor (five-foundation) structure with international subsamples and (2) control for demographics. It is possible, however, that the participants employed in the MFQ's foundational work may be meaningfully different from those used in subsequent research or the general population. Indeed, Iurino and Saucier (2020), after having failed to adequately establish measurement invariance in 23 of 27 countries where they administered the MFQ, point out that their samples were compensated monetarily rather than through "delivery of feedback" (p. 370) and significantly smaller (i.e. < 1,000). Representativeness is important in scale development, as "[t]he patterns of association among items might reflect unusual attributes shared among sample members but rare in the broader community. In other words, the groupings of interrelated items that emerge (e.g. from a factor analysis) may be atypical" (e.g. De Vellis, 2003). We do not intend to argue that the MFQ is flawed as a result of a volunteer sample being employed in its validation. However, research on samples drawn from populations different from those in J. Graham et al. (2011) did not fully replicate the five-factor structure, and showed some other differences in the scale's performance (Davis et al., 2016). Similarly, because reliability should be thought of as a characteristic of a particular dataset's or sample's performance on a scale (Streiner, 2003), a systematic examination of the MFQ in other samples would be of benefit to the

wider research community to better understand population-specific item functionality and psychometric properties. 


\section{Method}

\section{Sample}

A Google Scholar (http://scholar.google.com) search for the exact phrase "Moral Foundations Questionnaire" was conducted beginning at approximately 1 a.m. U.S. Eastern Daylight Time on March 16, 2017. Google Scholar was used for the search in an effort to identify the largest and most comprehensive sample possible, including unpublished manuscripts, theses and dissertations, and other documents that might not be captured by searches of specific academic databases. The precise phrase, "Moral Foundations Questionnaire" was used in an effort to identify documents that specifically used the questionnaire instrument rather than documents that referred to work related to moral foundations theory but included no use of the MFQ instrument. Results including only a citation but no readable document were excluded using the Google Scholar search engine's settings.

The initial search generated a list of 539 documents. Of these, 18 were duplicates, 84 documents could not be accessed for coding, and a further 40 were not written in English. Of the remaining 397 documents, coding revealed that 116 did not include analyses of MFQ items despite mentioning the MFQ. All of the 281 remaining documents included at least data for MFQ items from at least one sample, and some included MFQ data for two or more samples (multiple studies or multiple samples analyzed separately in a study). In the 281 documents using at least one sample with MFQ data, 358 samples with MFQ data were identified. In cases where a document contained MFQ data for more than one distinct sample, each sample was coded as a separate case in the data set. Uses of the same sample across multiple papers were retained in the dataset but excluded from any analyses reported here, leaving 344 unique research samples of MFQ item data (see Figure 1). Document information including title, authors(s), publication venue, publication venue title, year of publication, DOI, and number of citations identified by Google Scholar at the time of the search was recorded for each case from the Google Scholar search results and document files.

\section{Coded Variables}

MFQ version. Each research sample was coded for which version of the MFQ was used. We coded for the recognized MFQ-40, MFQ-30, MFQ-20. Variations on the forms that included or excluded "catch" items were also included in the coding. Any other deviations from the original items such as eliminating items, reconstituting subscales, or changing directions were coded as customized versions of the MFQ. Coders also indicated whether there was not enough information provided in the study to determine which version of the MFQ was employed. Type of reliability measure. Each research sample was coded for what reliability measure was used in the study (e.g., Cronbach's Alpha), or if a measure was not used or reported.

Overall MFQ reliability. Each research sample was coded for whether a reliability coefficient was reported for an overall measure of the MFQ questionnaire, and if so what coefficient was reported.

Subscale use. Each research sample was coded for whether each of the five MFQ subscales (care/harm, fairness/cheating, loyalty/betrayal, authority/subversion, and sanc- 

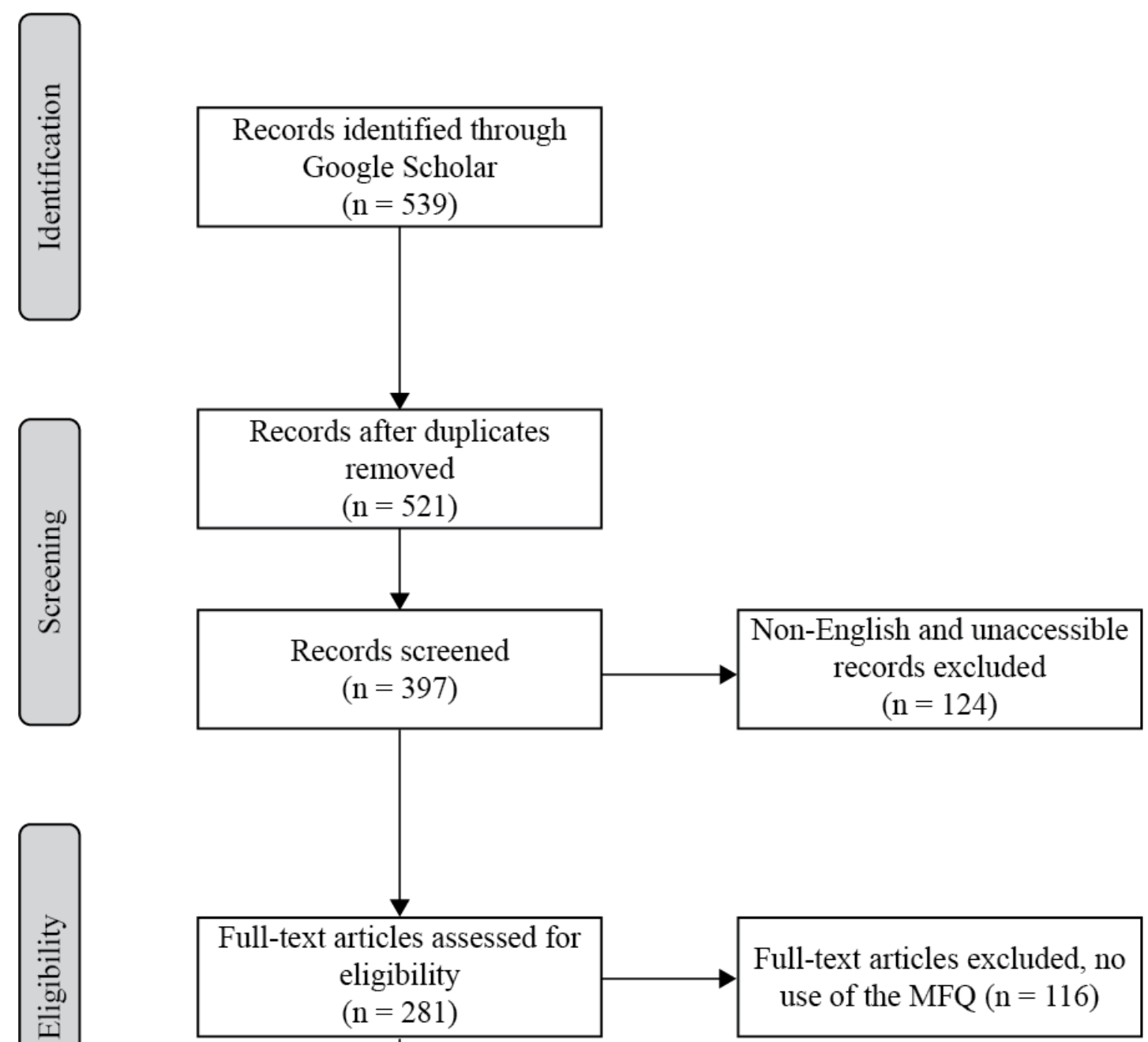

removed

$(\mathrm{n}=521)$
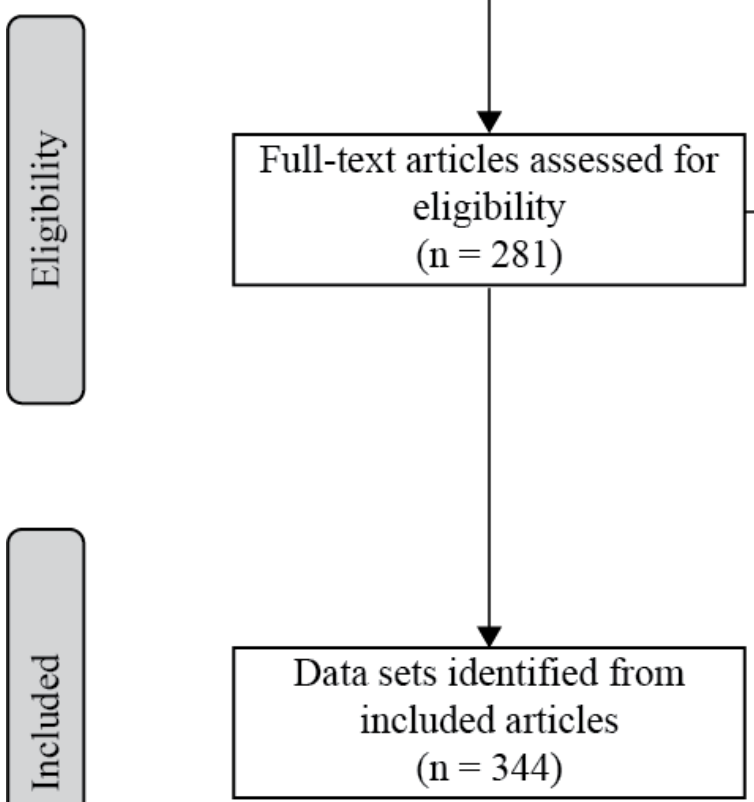

use of the MFQ $(n=116)$

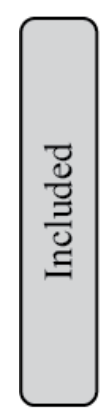

Data sets identified from ncluded articles

$\mathrm{n}=344)$

Figure 1. PRISMA flow diagram for identifying and screening articles for inclusion. 
tity/degradation) were used in the sample.

Subscale reliability. Each research sample was coded for whether a reliability coefficient was reported for each of the five MFQ subscales (care/harm, fairness/cheating, loyalty/betrayal, authority/subversion, and sanctity/degradation), and if so what coefficient was reported.

Measures not used in analysis. Initially, each research sample was coded for whether factor analysis was used to assess subscales in addition to reliability measures, and if so what rotation method was used, but these measures were not included in analyses here due to low prevalence of such instances and low intercoder reliability in an initial review of coded data. All measures and exclusions in the study are otherwise reported.

\section{Coding and Reliability}

The 539 initially identified documents were divided at random among two graduate student coders, with each coder assigned 298 documents including a randomly determined coding overlap of 57 cases $(19.1 \%)$ for assessment of intercoder reliability (indicated by Scott's $\pi$, see Table 1) $)^{1}$. Coders were not aware of which cases were included in the coding overlap.

Table 1

Reliability of the coding.

\begin{tabular}{llc}
\hline Coding & & $\begin{array}{c}\text { Reliability } \\
\text { (Scott's } \pi \text { ) }\end{array}$ \\
\hline Primary language & & 1.00 \\
Use of MFQ & & .79 \\
Reliability measure & & .78 \\
Overall MFQ reliability & & 1.00 \\
Care/Harm subscale: & $\ldots$ used & .84 \\
& $\ldots$ reliability & .97 \\
Fairness/Cheating subscale: & $\ldots$ used & .84 \\
& $\ldots$ reliability & .94 \\
Loyalty/Betrayal subscale: & $\ldots$ used & .81 \\
& $\ldots$ reliability & .94 \\
Authority/Subversion subscale: & $\ldots$ used & .84 \\
& $\ldots$ reliability & .94 \\
Sanctity/Degradation subscale: & $\ldots$ used & .87 \\
& $\ldots$ reliability & .94 \\
\hline
\end{tabular}

Later, two researchers coded for MFQ version used $(\pi=.86)$. The same researchers also coded for whether articles mentioned efforts to maintain quality responses. Individual data sets were coded for whether no such screening procedures were mentioned, removing

\footnotetext{
${ }^{1}$ Initially, three coders were recruited for coding, with assignments divided across all three coders, but preliminary analysis of coded data suggested inconsistent familiarity with identifying reliability measures across coders. As a result, one coder ceased coding work, with all of that coder's cases randomly assigned across the other two coders. The two coders who completed the final analyses completed all coding decisions without access to the erstwhile third coder's preliminary work.
} 
participants who answered questions in a random manner, or any other data quality control was employed $(\pi=.85)$.

\section{Additional Measures}

Publication type was coded (e.g. journal, dissertation, thesis, conference proceeding). Sample size and participant sample source were also recorded for each study, with the latter indicating whether a study used one of the prominent large, volunteer pools from yourmorals.org, Project Implicit, or Personality Lab. All other collection methods (e.g. department pools, class recruitment, MTurk) were collapsed.

\section{Results}

\section{MFQ Version}

Among the 344 research samples including data for a version of the MFQ, the most common identified version of the MFQ used was the MFQ-30, which was used in $54.4 \%$ ( $n$ $=187)$ of the samples. The MFQ20 was used in $7.8 \%(n=27)$ of the samples, and the MFQ-40 or equivalent was used in $1.7 \%(n=6)$ of the samples. Customized or altered versions of the MFQ were used in $15.4 \%(n=53)$ of the samples and $20.6 \%(n=71)$ were not described in sufficient detail in order to establish the specific form used.

\section{Type of Reliability Measure}

For $61.0 \%(n=210)$ of the samples, Cronbach's alpha was reported as a measure of reliability. The only other measure reported was correlation, which was used for $0.9 \%$ ( $n$ $=3)$ of the samples, while $38.1 \%(n=131)$ of the samples did not report a measure of reliability for MFQ items.

\section{Subscale Reliability}

In summary, the average Cronbach's alpha of most MFQ subscales was below .70, except for the Sanctity/Degradation subscale as it universally produced average alphas above .70 (see Table 1). Considering the four other subscales, the longer MFQ-40 produced an average alpha above .70 on the Care/Harm and Loyalty/Betrayal subscales, probably due to the larger amount of items. The MFQ-30 currently recommended for use on moralfoundations.org tended to produce lower average alphas (with even lower values for the MFQ-20 short form).

Although scale reliability is a characteristic of data, we also report scores weighted by sample size per Yin and Fan's (2000) recommendation since, as they claim, the size of a sample can impact the stability of a statistical estimate. Weighting the statistics by sample size can therefore reduce bias introduced by volatile estimates from smaller samples. This weighting excludes the one Pearson's correlation reported. Although larger samples should not affect reliability scores systematically, they provide greater precision to the internal consistency estimates than smaller samples. However, the difference between weighted and unweighted Cronbach's alphas was overall negligible. The MFQ-30 subscales generally had average alphas above .60 using the unweighted data, but using weighted data did show average alphas above .70 on the loyalty/betrayal and authority/subversion subscales. 
A small number of studies $(n=18)$ that used either yourmorals.org or Project Implicit to collect data account for a disproportionate number of the total participants (81.5\%). Since these large, volunteer data collection sources are unavailable to researchers generally, we also report weighted means excluding these studies and again exclusively drawing from these large data sets so that researchers who are using either of the two types of populations can have a more accurate understanding of the levels of reliability they may be able to expect from their populations. Although we do not report the results here, our data set also includes coding for publication venue and screening procedures employed, which can be used for moderator analysis of subscale reliabilities.

\section{Overall MFQ Reliability}

For $95.35 \%$ of the samples, no overall MFQ reliability score was reported. Among the samples $(n=16)$ for which an overall reliability score was reported, the mean reliability was .75 $(\mathrm{SD}=.09)$.

Table 2

Weighted and Unweighted Means and Standard Deviations for Subscale Reliabilities by Test Form.

\begin{tabular}{|c|c|c|c|c|c|}
\hline Foundation & MFQ Form & $\begin{array}{c}M_{\alpha}(\mathrm{SD}) \\
\mathrm{N}\end{array}$ & $\begin{array}{c}\text { Weighted } \\
\text { by } \mathrm{N}\end{array}$ & $\begin{array}{c}\text { Weighted by } \\
\mathrm{N} \text {, excluding } \\
\text { large samples } \\
M_{\alpha}(\mathrm{SD}) \\
\mathrm{N}\end{array}$ & $\begin{array}{c}\text { Weighted by } \\
\mathrm{N} \text {, exclusively } \\
\text { large samples } \\
M_{\alpha}(\mathrm{SD}) \\
\mathrm{N}\end{array}$ \\
\hline \multirow{12}{*}{ Care/Harm } & Total & $.65(.10)$ & $.68(.05)$ & $.65(.09)$ & $.69(.02)$ \\
\hline & & 146 & 337,902 & 56,869 & 280,223 \\
\hline & MFQ-20 & $.57(.07)$ & $.56(.06)$ & $.56(.06)$ & - \\
\hline & & 10 & 9,035 & 9,035 & - \\
\hline & MFQ-30 & $.66(.08)$ & $.69(.03)$ & $.66(.07)$ & $.69(.02)$ \\
\hline & & 85 & 309,767 & 33,369 & 276,398 \\
\hline & MFQ-40 & $.77(\mathrm{~N} / \mathrm{A})$ & $.77(\mathrm{~N} / \mathrm{A})$ & $.77(\mathrm{~N} / \mathrm{A})$ & - \\
\hline & & 1 & 286 & $.68(.13)$ & - \\
\hline & Customized & $.65(.15)$ & $.67(.12)$ & 8467 & $.62(\mathrm{~N} / \mathrm{A})$ \\
\hline & & 28 & 10,080 & 6,013 & 1,613 \\
\hline & Unspecified & $.65(.10)$ & $.68(.07)$ & $.67(.09)$ & $.71(\mathrm{~N} / \mathrm{A})$ \\
\hline & & 22 & 7,924 & 5,712 & 2,212 \\
\hline \multirow{8}{*}{$\begin{array}{l}\text { Fairness/ } \\
\text { Cheating }\end{array}$} & Total & $.65(.10)$ & $.65(.04)$ & $.66(.08)$ & $.64(.02)$ \\
\hline & & 151 & 319,665 & 62,808 & 256,857 \\
\hline & MFQ-20 & $.61(.14)$ & $.64(.09)$ & $.64(.09)$ & - \\
\hline & & 12 & 9,712 & 9,712 & - \\
\hline & MFQ-30 & $.65(.08)$ & $.64(.03)$ & $.65(.07)$ & $.64(.02)$ \\
\hline & & 87 & 290,272 & 37,240 & 253,032 \\
\hline & MFQ-40 & $.66(\mathrm{~N} / \mathrm{A})$ & $.66(\mathrm{~N} / \mathrm{A})$ & $.66(\mathrm{~N} / \mathrm{A})$ & - \\
\hline & & 1 & 286 & 286 & - \\
\hline
\end{tabular}




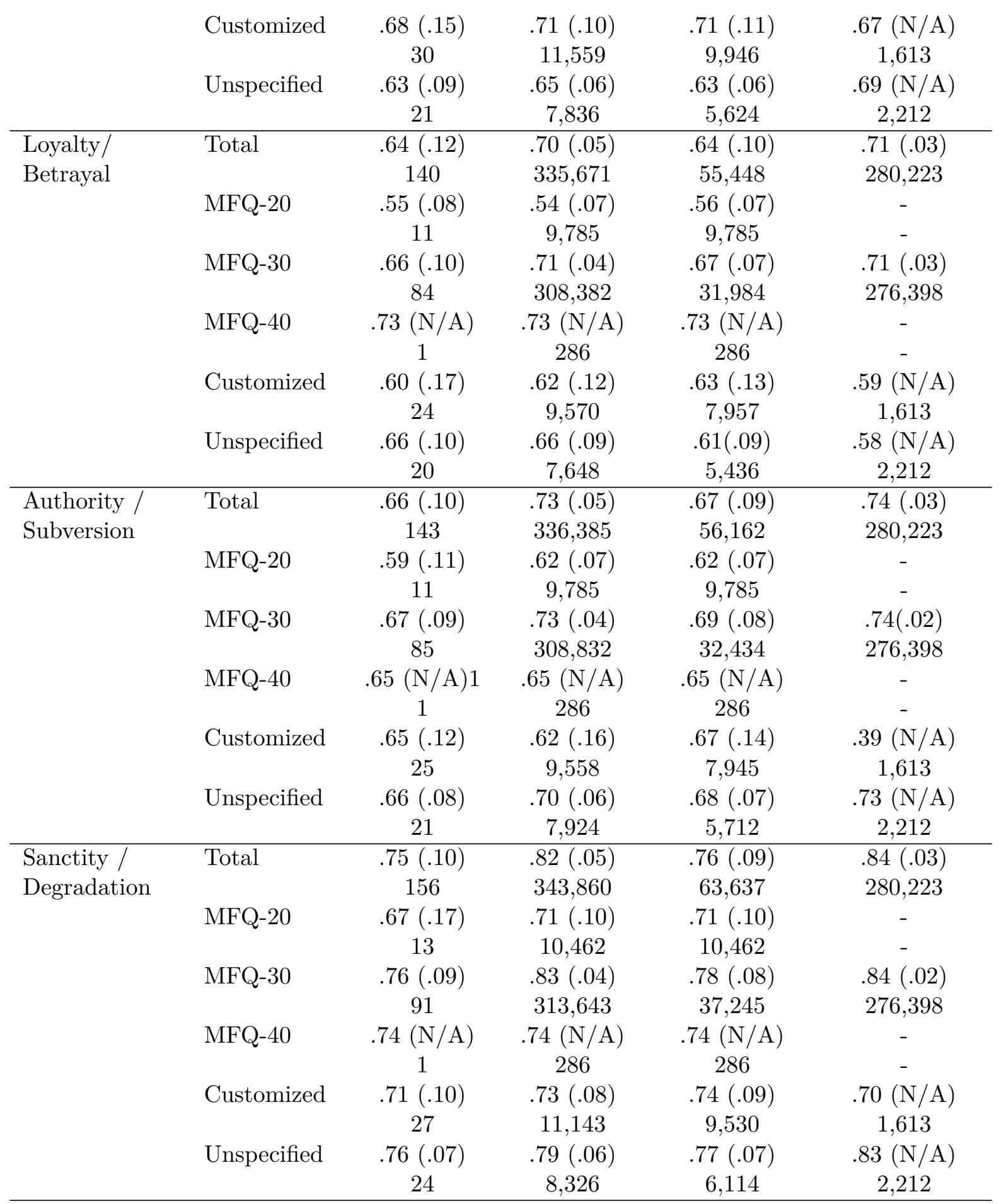

Note. Where no SD is provided, only one case is reported.

The $\mathrm{N}$ for unweighted columns is the number of samples that were used to calculate each mean. The $\mathrm{N}$ for weighted columns is the number of participants in that were used to calculate the weighted means. 


\section{Discussion}

The current study sought to examine the general reliability of the MFQ in its various forms. Our analysis suggests that reporting practices related to reliability are generally a problem in studies using the MFQ, with over one third of all studies failing to report any measure of reliability. All but one of those studies in which reliability was reported used Cronbach's alpha to indicate reliability. This is in line with similar past efforts to assess internal consistency and reliability. Authors should report reliability coefficients even when the MFQ is not the primary focus of their investigation because doing so would assist others in making sound decisions about the soundness of the MFQ, and assist meta-analytic research efforts like this one.

In J. Graham et al.'s (2011) original work, three of the five scales surpassed what researchers, for better or for worse, generally consider an acceptable Cronbach's alpha cutoff of .70 (Nunnally, 1978). Our findings indicate that the internal consistency of the MFQ's subscales was almost across the board slightly lower than in J. Graham et al.'s (2011) scale validation study, with the exception of the fairness/cheating subscale, which on average had a slightly higher Cronbach's alpha than J. Graham et al. (2011). Researchers should calibrate their expectations for the MFQ's performance accordingly, and take into account these implications as they plan their study, particularly with regards to their sampling and statistical power.

Given that alpha is a point estimate, it is possible that larger sample sizes are able to provide a more accurate assessment of the MFQ's internal consistency. When we weighted the average alpha by sample size, we observed almost universally that the average alpha either remains the same or increases slightly. What is not clear from our analyses here is whether the slightly higher estimates are the result of a more accurate assessment of the scales' internal consistency or some characteristic of the populations from which the larger samples were drawn.

A logical next step then would be to retool the MFQ items to create more reliable responses from participants. In the scale's design, J. Graham et al. (2009) claimed that they were attempting to balance internal consistency with content validity - the ability for a scale to exhaustively measure a concept (Bannigan \& Watson, 2009). Yet, building a scale that samples from each aspect of a concept should result in a scale where individual items are highly interrelated (Henson, 2001). Furthermore, internal consistency and content validity are not inherently at odds with one another. While we cannot say what it is about the individual items that makes the subscales susceptible to error, given the scale's widespread use it would certainly be a worthwhile investment for the field at large to improve it.

The overall low internal consistency has implications beyond merely the degree of error relative to true variance in its items. We would be remiss not to contextualize our examination of reliability within a growing body of work on the validity of measures in the field more generally and the MFQ specifically. As Hussey and Hughes (2018) found in their analysis of several popular scales, $89 \%$ of the measures met or exceeded $\alpha=.70$, but only $4 \%$ also showed acceptable indicators of validity in more rigorous tests of measurement validity. With the exception of the sanctity-degradation subscale, the MFQ's scales generally 
do not meet that threshold. In light of Hussey and Hughes's (2018) work and our own findings presented here, one might hypothesize that the MFQ similarly lacks measurement invariance, which is supported by Iurino and Saucier's (2020) inability to demonstrate adequate measurement invariance in a cross-cultural context. Given that measurement validity is a precursor to making valid claims based on observations, our own observations, in line with, for example, Iurino and Saucier (2020) and Davis et al. (2016), might serve as a call to revisit the evidentiary basis for MFT more broadly.

\section{Limitations and Future Work}

Naturally, this study provides only a partial answer to the question of the MFQ's validity. Describing the conditions under which the MFQ would be considered valid, and then to test whether each of those conditions is true, would be a massive undertaking, even beyond the impressively large dataset by J. Graham et al. (2011). First, a wealth of resources already exist that can describe the necessary conditions in order for a scale to be a valid measure of a construct (Borsboom, 2005 Markus \& Borsboom, 2013 Nunnally, 1967, 1978, Zumbo \& Chan, 2014). A more exhaustive examination of the scale might seek to determine whether the MFQ possesses content, discriminant, and convergent validity, for example, in addition to testing the reliability of the subscales. We agree that such an examination would be warranted, especially given the findings of our current work, but given the poor state of the field in reporting more detailed indicators of validity (see Flake et al., 2017), even had we sought out to summarize the MFQ's performance on indicators other than alpha, we would have likely been unsuccessful.

This brings us to our second point, which we make out of an abundance of caution. We chose to examine a necessary component of validity by examining the scale's reliability, a necessary requirement to validity (Nunnally, 1967, 1978). Although we consider our findings evidence for the possibility that the MFQ may lack some form of validity, we recognize that internal consistency (indicated by Cronbach's alpha) is only one component of psychometric reliability, and that the MFQ may well be a reliable instrument despite consistently low internal consistency. However, there is a dearth of attempts to demonstrate the MFQ's reliability (beyond reported Cronbach's alpha). Certainly, others may reasonably disagree with our concerns of the moderate Cronbach's alpha scores achieved across the literature, and we would welcome a principled debate if substantially higher internal consistency (a) can be reasonably expected, and (b) may even be desirable. We also encourage MFT scholars to include one or more alternative indicators of the psychometric properties of their scales in their reporting of results on the MFQ, such as McDonald's omega. Practical guides and scripts to facilitate this have been made available for R, SAS, SPSS, Mplus, and AMOS (Dunn et al., 2014 Hayes \& Coutts, 2020). While some information on reliability is certainly better than none, alpha has been widely critiqued as an inappropriate measure for scale reliability (McNeish, 2018; McNeish et al., 2018; Raykov, 1997, e.g.) in part because it assumes that scale items are at least essentially tau-equivalent-that is to say that the scale is unidimensional, all items on the factor have the same weight, and that residuals are invariant between items.

Violating this assumption leads to underestimating alpha and reliability (J. M. Graham, 2006). However, Raykov and Marcoulides (2019) point out several critical errors in 
McNeish's (2018) article, but it is not our purpose here to determine whether alpha is an appropriate measure of interitem correlation or reliability. Rather, because alpha is generally the most common indicator of reliability and this was also this case in our analysis, future work should first reexamine the validity of the individual MFQ subscales as valid measures for moral foundations. Second, subsequent work can determine the specific properties of the MFQ items such as whether they are congeneric, which is to say that they measure the same unidimensional latent construct but with varying degrees of precision and error. Alpha may underestimate reliability if the scale is not congeneric and additional work will be needed to accurately capture its general reliability since it is difficult to determine the extent of the underestimation (J. M. Graham, 2006 Lord \& Novick, 1968). Answering these basic questions would aid future scholars in interpreting the findings of our work here. In the case of the MFQ, the underestimation of alpha may be significant as the underestimation is greater when scales have fewer items (Raykov, 1997).

We examined published studies that use the MFQ, but we collected a dataset that is likely to be systematically different from the universe of research done with the MFQ because, as we stated earlier, many of the studies that used the MFQ did not report on its reliability, and because this literature, like any other, may be shaped by publication bias (Dickersin, 1990, 2005, see). At this point, we do not know whether the MFQ's reliability in unpublished studies or in published studies that have not reported its reliability is systematically different from those examined here. Researchers should not conclude that our findings adequately capture the true average reliabilities of the MFQ scales for these reasons. The general alpha levels of the MFQ subscales could very well be lower than what we have found here. We purposely chose to conduct our search on Google Scholar, which indexes a large number of sources beyond academic journals, and were able to include a relatively large number of studies (published and unpublished) in the content analysis. However, researchers have expressed concerns about using Google Scholar as principal search system for systematic reviews due to issues with precision, recall, and reproducibility (Gusenbauer \& Haddaway, 2020). Further, given the many translations of the MFQ into other languages, it is conceivable that there are studies published in other languages which we might have missed with our English-language search term. These studies could be of particular importance as they are more likely to be conducted with samples of populations other than those in English-language publications generally, and the MFQ's foundational work (J. Graham et al., 2011) specifically.

Future examinations of the body of published literature could also examine the scales' performance over time, the relationship between a study's citations and reliability - perhaps studies that are more methodologically sound are cited more often have made better use of the MFQ. Additional work could also be done on whether the MFQ, due to researchers' diminished power to detect statistically significant relationships, is leading to a propensity of barely significant studies.

\section{Open Practices and ethics}

Our data and scripts can be accessed on the Open Science Framework (https://osf.io/hsq39/). This article adheres to the APA code of ethics. The authors have no conflicts of interest to declare. 


\section{References}

Bannigan, K., \& Watson, R. (2009). Reliability and validity in a nutshell. Journal of Clinical Nursing, 18(23), 3237-3243. https://doi.org/10.1111/j.1365-2702.2009.02939.x

Barry, A. E., Chaney, B., Piazza-Gardner, A. K., \& Chavarria, E. A. (2014). Validity and reliability reporting practices in the field of health education and behavior. Health Education \& Behavior, 41(1), 12-18. https://doi.org/10.1177/1090198113483139

Borsboom, D. (2005). Measuring the mind: Conceptual issues in contemporary psychometrics. Cambridge, UK, Cambridge University Press.

Davis, D. E., Rice, K., Van Tongeren, D. R., Hook, J. N., DeBlaere, C., Worthington, E. L., \& Choe, E. (2016). The moral foundations hypothesis does not replicate well in Black samples. Journal of Personality and Social Psychology, 110(4), e23-e30. https://doi.org/10.1037/pspp0000056

De Vellis, R. F. (2003). Scale development: Theory and applications (2nd ed.). Thousand Oaks, CA, Sage.

Dickersin, K. (1990). The existence of publication bias and risk factors for its occurrence. JAMA, 263(10), 1385-1389. https://doi.org/10.1001/jama.1990.03440100097014

Dickersin, K. (2005). Publication bias: Recognizing the problem, understanding its origins and scope, and preventing harm (H. R. Rothstein, A. J. Sutton, \& M. Borenstein, Eds.). In H. R. Rothstein, A. J. Sutton, \& M. Borenstein (Eds.), Publication bias in meta-analysis: Prevention, assessment and adjustments. Chichester, UK, Wiley. https://doi.org/10.1002/0470870168.ch2

Drost, E. A. (2011). Validity and reliability in social science research. Education Research and Perspectives, 38(1), 105-123.

Dunn, T. J., Baguley, T., \& Brunsden, V. (2014). From alpha to omega: A practical solution to the pervasive problem of internal consistency estimation. British Journal of Psychology, 105(3), 399-412. https://doi.org/10.1111/bjop.12046

Elman, J. L., Bates, E., Johnson, M. H., Karmiloff-Smith, A., Parisi, D., \& Plunkett, K. (1996). Rethinking innateness: A connectionist perspective on development. Cambridge, MA, MIT Press.

Flake, J. K., Pek, J., \& Hehman, E. (2017). Construct validation in social and personality research. Social Psychological and Personality Science, 8(4), 370-378. https://doi. org/10.1177/1948550617693063

Gilligan, C. (1982). In a different voice: Psychological theory and women's development. Cambridge, MA, Harvard University Press.

Gilligan, C., \& Wiggins, G. (1987). The origins of morality in early childhood relationships (J. Kagain \& S. Lamb, Eds.). In J. Kagain \& S. Lamb (Eds.), The emergence of morality in young children. Chicago, IL, University of Chicago Press.

Graham, J. M. (2006). Congeneric and (essentially) tau-equivalent estimates of score reliability. Educational and Psychological Measurement, 66(6), 930-944. https://doi. org $/ 10.1177 / 0013164406288165$

Graham, J., \& Haidt, J. (2012). Sacred values and evil adversaries: A moral foundations approach (M. Mikulincer \& P. R. Shaver, Eds.). In M. Mikulincer \& P. R. Shaver (Eds.), The social psychology of morality: Exploring the causes of good and evil. 
Washington, DC, American Psychological Association. https://doi.org/10.1037/ 13091-001

Graham, J., Haidt, J., \& Nosek, B. A. (2009). Liberals and conservatives rely on different sets of moral foundations. Journal of Personality and Social Psychology, 96(5), 10291046. https://doi.org/10.1037/a0015141

Graham, J., Nosek, B. A., \& Haidt, J. (2012). The moral stereotypes of liberals and conservatives: Exaggeration of differences across the political spectrum. PLoS ONE, 7(12), e50092. https://doi.org/10.1371/journal.pone.0050092

Graham, J., Nosek, B. A., Haidt, J., Iyer, R., Koleva, S., \& Ditto, P. H. (2011). Mapping the moral domain. Journal of Personality and Social Psychology, 101(2), 366-385. https://doi.org/10.1037/a0021847

Gray, K., \& Keeney, J. E. (2015a). Disconfirming Moral Foundations Theory on its own terms. Social Psychological and Personality Science, 6(8), 874-877. https://doi. org/10.1177/1948550615592243

Gray, K., \& Keeney, J. E. (2015b). Impure or just weird? Scenario sampling bias raises questions about the foundation of morality. Social Psychological and Personality Science, 6 (8), 859-868. https://doi.org/10.1177/1948550615592241

Gusenbauer, M., \& Haddaway, N. R. (2020). Which academic search systems are suitable for systematic reviews or meta-analyses? Evaluating retrieval qualities of Google Scholar, PubMed, and 26 other resources. Research Synthesis Methods, 11(2), 181217. https://doi.org/10.1002/jrsm.1378

Haidt, J. (2017). Moral foundations theory. http://www.moralfoundations.org

Haidt, J., \& Graham, J. (2007). When morality opposes justice: Conservatives have moral intuitions that liberals may not recognize. Social Justice Research, 20(1), 98-116. https://doi.org/10.1007/s11211-007-0034-z

Haidt, J., \& Graham, J. (2009). Planet of the Durkheimians, where community, authority, and sacredness are foundations of morality (J. T. Jost, A. C. Kay, \& H. Thorisdottir, Eds.). In J. T. Jost, A. C. Kay, \& H. Thorisdottir (Eds.), Social and psychological bases of ideology and system justification. New York, NY, Oxford University Press. https://doi.org/10.1093/acprof:oso/9780195320916.003.015

Haidt, J., Graham, J., \& Joseph, C. (2009). Above and below left-right: Ideological narratives and moral foundations. Psychological Inquiry, 20(2-3), 110-119. https://doi. org $/ 10.1080 / 10478400903028573$

Haidt, J., \& Joseph, C. (2004). Intuitive ethics: How innately prepared intuitions generate culturally variable virtues. Daedalus, 133(4), 55-66. https:// doi.org/10.1162/ 0011526042365555

Haidt, J., \& Joseph, C. (2008). The moral mind, In The innate mind. New York, NY, Oxford University Press. https://doi.org/10.1093/acprof:oso/9780195332834.003.0019

Haidt, J., \& Joseph, C. (2011). How moral foundations theory succeeded in building on sand: A response to Suhler and Churchland. Journal of Cognitive Neuroscience, 23(9), 2117-2122. https://doi.org/10.1162/jocn.2011.21638

Hatemi, P. K., Crabtree, C., \& Smith, K. B. (2019). Ideology justifies morality: Political beliefs predict moral foundations. American Journal of Political Science, 63(4), 788806. https://doi.org/10.1111/ajps.12448 
Hayes, A. F. (2005). Statistical methods for communication science. New York, NY, Routledge.

Hayes, A. F., \& Coutts, J. J. (2020). Use omega rather than Cronbach's alpha for estimating reliability. But... Communication Methods and Measures, 14(1), 1-24. https://doi. org/10.1080/19312458.2020.1718629

Hedge, C., Powell, G., \& Sumner, P. (2018). The reliability paradox: Why robust cognitive tasks do not produce reliable individual differences. Behavior Research Methods, 50(3), 1166-1186. https://doi.org/10.3758/s13428-017-0935-1

Henson, R. K. (2001). Understanding internal consistency reliability estimates: A conceptual primer on coefficient alpha. Measurement and Evaluation in Counseling and Development, 34 (3), 177-189. https://doi.org/10.1080/07481756.2002.12069034

Hussey, I., \& Hughes, S. (2018). Hidden invalidity among fifteen commonly used measures in social and personality psychology. https://doi.org/10.31234/osf.io/7rbfp

Iurino, K., \& Saucier, G. (2020). Testing measurement invariance of the Moral Foundations Questionnaire across 27 countries. Assessment, $27(2), 365-372$. https://doi.org/10. $1177 / 1073191118817916$

Iyer, R., Graham, J., Koleva, S., Ditto, P., \& Haidt, J. (2010). Beyond identity politics: Moral psychology and the 2008 democratic primary. Analyses of Social Issues and Public Policy, 10(1), 293-306. https://doi.org/10.1111/j.1530-2415.2010.01203.x

John, I. D. (1992). Statistics as rhetoric in psychology. Australian Psychologist, 27(3), 144149. https://doi.org/10.1080/00050069208257601

John, O. P., \& Soto, C. J. (2007). The importance of being valid: Reliability and the process of construct validation. (R. W. Robins, R. C. Fraley, \& R. F. Krueger, Eds.). In R. W. Robins, R. C. Fraley, \& R. F. Krueger (Eds.), Handbook of research methods in personality psychology. New York, NY, Guilford Press.

Jost, J. T., Glaser, J., Kruglanski, A. W., \& Sulloway, F. J. (2003). Political conservatism as motivated social cognition. Psychological Bulletin, 129(3), 339-375. https://doi. org/10.1037/0033-2909.129.3.339

Kohlberg, L. (1969). Stage and sequence: The cognitive development approach to socialization (D. A. Goslin, Ed.). In D. A. Goslin (Ed.), Handbook of socialization theory. Rand McNally.

Koleva, S. P., Graham, J., Iyer, R., Ditto, P. H., \& Haidt, J. (2012). Tracing the threads: How five moral concerns (especially purity) help explain culture war attitudes. Journal of Research in Personality, 46(2), 184-194. https://doi.org/10.1016/j.jrp.2012.01.006

Lord, F. M., \& Novick, M. R. (1968). Statistical theories of mental test scores. Reading, MA, Addison-Wesley.

Marcus, G. (2004). The birth of the mind. New York, NY, Basic.

Markus, K. A., \& Borsboom, D. (2013). Frontiers of test validity theory: Measurement, causation, and meaning. New York, NY, Routledge.

McNeish, D. (2018). Thanks coefficient alpha, we'll take it from here. Psychological Methods, 23(3), 412-433. https://doi.org/10.1037/met0000144

McNeish, D., An, J., \& Hancock, G. R. (2018). The thorny relation between measurement quality and fit index cutoffs in latent variable models. Journal of Personality Assessment, $100(1), 43-52$. https://doi.org/10.1080/00223891.2017.1281286 
Meier, S. T. (1994). The chronic crisis in psychological measurement and assessment: A historical survey. San Diego, CA, Academic Press.

Meier, S. T., \& Davis, S. R. (1990). Trends in reporting psychometric properties of scales used in counseling psychology research. Journal of Counseling Psychology, 37(1), 113-115. https://doi.org/10.1037/0022-0167.37.1.113

Meyer, P. (2010). Reliability. New York, NY, Oxford University Press.

Miller, M. B. (1995). Coefficient alpha: A basic introduction from the perspectives of classical test theory and structural equation modeling. Structural Equation Modeling: A Multidisciplinary Journal, 2(3), 255-273. https://doi.org/10.1080/ 10705519509540013

Niemi, R. G., Carmines, E. G., \& McIver, J. P. (1986). The impact of scale length on reliability and validity. Quality and Quantity, 20(4). https://doi.org/10.1007/ BF00123086

Nimon, K., Zientek, L. R., \& Henson, R. K. (2012). The assumption of a reliable instrument and other pitfalls to avoid when considering the reliability of data. Frontiers in Psychology, 3. https://doi.org/10.3389/fpsyg.2012.00102

Nunnally, J. C. (1967). Psychometric theory. New York, NY, McGraw-Hill.

Nunnally, J. C. (1978). Psychometric theory (2nd ed.). New York, NY, McGraw-Hill.

Nunnally, J. C., \& Bernstein, I. H. (1994). Psychometric theory (3rd ed.). New York, NY, McGraw-Hill.

Pinker, S. (2002). The blank slate. The modern denial of human nature. New York, NY, Viking.

Raykov, T. (1997). Scale reliability, Cronbach's coefficient alpha, and violations of essential tau-equivalence with fixed congeneric components. Multivariate Behavioral Research, 32(4), 329-353. https://doi.org/10.1207/s15327906mbr3204\{\_\}2

Raykov, T., \& Marcoulides, G. A. (2019). Thanks coefficient alpha, we still need you! Educational and Psychological Measurement, 79(1), 200-210. https://doi.org/10. $1177 / 0013164417725127$

Reichwein Zientek, L., Capraro, M. M., \& Capraro, R. M. (2008). Reporting practices in quantitative teacher education research: One look at the evidence cited in the AERA panel report. Educational Researcher, 37(4), 208-216. https://doi.org/10. 3102/0013189X08319762

Schmitt, N. (1996). Uses and abuses of coefficient alpha. Psychological Assessment, 8(4), 350-353. https://doi.org/10.1037/1040-3590.8.4.350

Sijtsma, K. (2009). On the use, the misuse, and the very limited usefulness of Cronbach's alpha. Psychometrika, 74 (1), 107-120. https://doi.org/10.1007/s11336-008-9101-0

Spearman, C. (1904). The proof and measurement of association between two things. International Journal of Epidemiology, 72-101. https://doi.org/10.1093/ije/dyq191

Streiner, D. L. (2003). Starting at the beginning: An introduction to coefficient alpha and internal consistency. Journal of Personality Assessment, 80(1), 99-103. https://doi. org/10.1207/S15327752JPA8001\{\_\}18

Suhler, C. L., \& Churchland, P. (2011). Can innate, modular "foundations" explain morality? Challenges for Haidt's Moral Foundations Theory. Journal of Cognitive Neuroscience, 23(9), 2103-2116. https://doi.org/10.1162/jocn.2011.21637 
Thompson, B. (2003). Understanding reliability and coefficient alpha, really (B. Thompson, Ed.). In B. Thompson (Ed.), Score reliability: Contemporary thinking on reliability issues. Thousand Oaks, CA, Sage. https://doi.org/10.4135/9781412985789.n1

Turiel, E. (1983). The development of social knowledge: Morality and convention. Cambridge, UK, Cambridge University Press.

Vacha-Haase, T., Ness, C., Nilsson, J., \& Reetz, D. (1999). Practices regarding reporting of reliability coefficients: A review of three journals. The Journal of Experimental Education, 67(4), 335-341. https://doi.org/10.1080/00220979909598487

Wrench, J. S., Thomas-Maddox, C., Peck Richmond, V., \& McCroskey, J. C. (2013). Quantitative research methods for communication: A hand-on approach (2nd ed.). New York, NY, Oxford University Press.

Yin, P., \& Fan, X. (2000). Assessing the reliability of Beck Depression Inventory scores: Reliability generalization across studies. Educational and Psychological Measurement, 60 (2), 201-223. https://doi.org/10.1177/00131640021970466

Zumbo, B. D., \& Chan, E. K. H. (Eds.). (2014). Setting the stage for validity and validation in social, behavioral, and health sciences: Trends in validation practices (Springer). New York, NY. 